\title{
Social Media, Grindr, and PrEP: Sexual health literacy for men who have sex with men in the Internet age
}

Gavin Goodwin, M.M., BMus

School of Information Studies, McGill University

\begin{abstract}
Despite continued improvements to HIV/AIDS treatment and awareness, HIV transmission rates remain high among men who have sex with men (MSM). Online consumer health information targeting high risk MSM through social media and geosocial networking (GSN) apps have shown to be successful HIV intervention strategies. This review article addresses (1) the efficacy and acceptance of delivering consumer health information about pre-exposure prophylaxis (PrEP) and HIV prevention through GSN apps, (2) the impact of online and social media communities in the discussion and delivery of information about PrEP and HIV interventions, and (3) on-going and possible future research and the role of information professionals.
\end{abstract}

\section{Introduction}

Throughout the ongoing struggle against the HIV/AIDS epidemic worldwide and with new advancements in treatment and prevention, higher incidences of infection and transmission of HIV continue to be reported among high-risk populations like men who have sex with men (MSM) [1]. Of the new HIV infections in Canada reported in 2018, 58\% were among gay, bisexual, or other MSM [2]. Health Canada reports an estimated 75,500 Canadians currently living with HIV and approximately $1 / 5$ of whom are currently undiagnosed or unaware of their HIV status [3]. Despite the overall decrease in HIV cases among industrialized nations, MSM rates remain high even with targeted health interventions for high risk groups, highlighting the difficulties in the transmission and propagation of credible and engaging consumer health information concerning HIV prevention and treatment [1].

Within the past decade, new biomedical interventions have changed the reality of many who are at risk of HIV infection through reducing the rate of transmission with strategies of treatment as prevention. These strategies include the use of antiretroviral drugs to achieve 
undetectable viral loads in HIV-positive individuals and the use of once-daily oral pre-exposure prophylaxis (PrEP) to prevent new infections in HIV-negative individuals.

First approved by the American Food \& Drug Administration in 2012, PrEP drugs like emtricitabine and tenofovir (commonly sold under the trade name Truvada) have the potential to be a turning point in the reduction of HIV transmission [4]. Current Canadian health guidelines propose the use of PrEP by those deemed to be at high risk of HIV infection, including men or trans women who report condomless sex with men, those who have other sexually transmitted infections (STI), those with high scores using risk assessment tools, anyone engaging in condomless sex with a non-treated HIV-positive partner, or those who share injection drug use equipment [5]. While clinical research about the effectiveness of PrEP is ongoing, current findings indicate a high degree of success (up to $99 \%$ effectiveness) in preventing the transmission of HIV when PrEP is taken daily [5]. Despite the mounting scientific evidence about the effectiveness of PrEP, uptake rates of the drug are slow, with only 1,774 HIV-negative people reported taking the drug in the United States between 2011 and 2013 [4]. Estimates by the AIDS Vaccine Advocacy Coalition place the current number of PrEP users in Canada in 2020 at approximately 4,700-5,200 people [6]. Concerns about the efficacy of the drug, cost and availability, side effects, and risk compensation concerning sexual behaviour are possible indicators for the slow uptake in the drug. Effective consumer health information is required to help assuage fears, counter misinformation, and spread awareness to increase uptake and maintain effective drug administration.

The current ubiquity of online information use and mobile phones has led electronic health (eHealth) and mobile health (mHealth) to be seen as viable and important vectors for the distribution of consumer health information in order to change behaviour, enhance treatment 
efficacy, increase patient knowledge, and improve clinical outcomes [7]. While smartphone applications have been developed with the goal of offering information about HIV and other STI transmission, the infrequent app downloads, inability to reach large numbers of high risk people, and overall poor reception and reviews [8] has led public health campaigns to use both social media platforms and popular geosocial networking (GSN) apps to reach high-risk MSM populations. GSN smartphone apps, (e.g., Grindr, Jack'd, Scruff, Growlr, etc.) allow MSM to connect through an online social platform to find sexual partners based on geographic proximity with these apps gaining popularity since their first emergence approximately 10 years ago [9]. The most popular of these services, Grindr, has reported over 2 million daily users worldwide [1] and due to their influence in interpersonal and sexual relationships among MSM, these apps present fruitful opportunities for communication and knowledge transfer concerning HIV prevention [10]. Likewise, the widespread use of social media platforms like Facebook, Twitter, and Instagram present possible mediums for the spread of HIV health information to MSM groups that are more difficult to reach, allowing repeated exposure to messaging and offering the possibility to disseminate information to large numbers of people at once [11].

This review article will discuss (1) the efficacy and acceptance of delivering consumer health information about PrEP and HIV prevention through GSN apps, (2) the impact of online and social media communities in the discussion and delivery of information about PrEP and HIV interventions, and (3) on-going and possible future research and the role of information professionals. 


\section{Literature Review}

The search strategy for this review included a survey of literature from the medical, information technology, and information science disciplines. The focus included HIV/AIDS treatment and prevention, consumer health information, internet health research, and evaluation of health information literacy. Inclusion of articles in the review required a focus on the use or implementation of PrEP for high risk populations, a study of GSN apps, or social media-based health information interventions, as well as a recent publishing date within the past 10 years. Specific search terms used included: geosocial networking apps (and related trade names), social media (and specific platforms), pre-exposure prophylaxis (and trademarked drug names), men who have sex with men, risk management and HIV/AIDS.

\section{Discussion}

\section{Geosocial Networking Apps and sexual health information}

Indications show the use of the internet by a large portion of the sexually active population, including rates as high as one third of adults looking for sexual partners online and also those using the internet tending to be more sexually active [12]. The use of GSN apps for MSM is also high, with some rates found as many as one in three MSM having used a GSN app, $85 \%$ of users logging on with daily frequency [13] and apps like Grindr having as many as 2 million daily active users globally [1]. Due to the multifunction use of GSN apps as methods of interpersonal interactions (both social and sexual), the frequent and often daily use by MSM, and their potential to reach traditionally underserved or hard-to-reach populations, they have been identified as useful mediums for the conveyance of health information and targeted sexual health interventions [9]. This includes targeting particularly important populations like MSM who are uncertain of their current HIV status or those with low self-efficacy in STI testing [8]. 
Additionally, GSN apps have been used as effective tools assessing the need for and interest in the implementation of PrEP programs in new regions [14], further strengthening the argument for their ability to reach high risk MSM and those interested in targeted consumer health information for HIV prevention.

\subsection{Acceptability of sexual health information dissemination using GSN apps}

The reception of sexual health information for MSM using GSN apps has been positive, with high overall acceptability $[8,15]$. Other encouraging trends show that these interventions are generally well received regardless of sociodemographic variables like race, ethnicity, age, income, education, or geographic location $[15,16]$. Community feedback describes favourably the incorporation of sexual health sections and advertisement banners within GSN apps, with the frequency of information delivery including both information actively sought out and weekly push alerts. $[9,15]$. Information concerning PrEP is among the most acceptable types of information offered through the apps, which offers encouraging evidence to continue health messaging through GSN apps in an effort to increase PrEP uptake, especially in rural or less centralized urban centres [15].

\subsection{Emerging recommendations and best practices for GSN app health messaging}

Ongoing research on the acceptability and effectiveness of GSN app sexual health messaging has highlighted several emerging trends and best practices which could be of use to consumer health providers and those designing similar targeted public health interventions. These recommendations include the ability to distinguish legitimate health information from spam or fraudulent messages, a focus on reaching populations outside the traditionally predominant gay social spaces and avoiding stigmatization of groups by solely targeting higher risk demographics like young MSM or racial or ethnic minorities [9,17]. Timeliness of 
information delivery is a noted benefit of GSNs, though information should not hinder the experience and enjoyment of meeting partners through GSN apps to maintain favourability among users [18]. Information offered should remain both engaging and sex positive, avoid overly clinical language, work to build a culture of precautionary social norms, and should be delivered by authoritative and trusted information sources [18].

The geolocation functionality of the apps offers great potential for providing geographically tailored information about local resources and HIV testing, though caution should be exercised to avoid the perception that too much personalization is intrusive and violates the privacy of users [18].

The encouraging feedback from users along with the emergence of recommended best practices for GSN sexual health messaging suggests the continued use and development of these HIV intervention strategies and further refinement of messaging and information tailored to this medium. Behaviour concerning HIV status and use of PrEP between app users is another consideration. Disclosing PrEP use or serostatus is an increasingly common practice among GSN app users and this information exchange factors into risk analysis concerning HIV transmission. This increase in app user PrEP use, awareness, and communication strengthens evidence that GSN apps offer an impactful intervention point to provide accurate and relevant consumer health information [19].

\section{Social media and sexual health information for MSM}

While GSN apps have a significant reach and impact among high risk MSM, they do not have the universality and ability to reach a broader range of people as does social media. The ubiquity of platforms like Facebook, Twitter, and Instagram allow sexual health information to reach more diverse populations while being cost effective and easily scaled-up, allowing 
repeated exposure to information with built-in user feedback, and to encourage direct engagement between information seekers and providers [11]. Possible communication mechanisms include crowd sourcing, pop-up ads, establishing virtual communities, online counselling, education and FAQs, and referral for STI testing [11]. There are also existing online communities centred around popular and online meme culture for the LGBTQ2IA+ community with millions of followers, demonstrating the large-scale possibilities for consumer health information dissemination when addressing the appropriate audiences [20].

Social media has also been highlighted by users as a medium of interest for delivery of sexual health information, especially among youth as it allows the exchange of information with peers and helps foster an interest in and engagement with their own sexual health [21]. The potential held by social media is important not only for the dissemination of sexual health information but also as a possible source of study and data for researchers looking to understand the information behaviour of those searching for, consuming, and sharing health information online.

\subsection{Facebook, Twitter, Instagram: how is the information used?}

Of the popular social media platforms available, Facebook, Twitter, and Instagram are commonly used in sexual health and HIV interventions online, with Instagram, Twitter, and Snapchat cited as of being particular use in reaching younger people [17]. Gaining an understanding of the content of the health information shared on social media can be of value to consumer health providers, showing how communities at high risk are engaging in HIV and PrEP-related communication. This includes if and how the information and sentiments being shared are aligned with those of public health priorities, as well as identifying unique and 
beneficial messages that could be amplified and propagated with support from health information professionals [22].

Studies analysing HIV-related hashtag trends on Twitter highlight that platform as being used primarily for the sharing of general health information and news rather than personal expression and discussion, as well as the limitations of targeting specific high risk groups due to the character limits of Tweets forcing a trade-off between target specificity and how informationrich the communication can be $[23,24]$. A similar analysis of visual content on Instagram found that most social media posts and discussion concerns infographics and stigma reduction about HIV and little about clinical interventions, PrEP use, or general public sexual health priorities [22]. Facebook is perhaps the most successful in both delivering and sharing sexual health information due to its participatory features and networking capability that helps foster solidarity and community, with great success found through the use of popular opinion leaders and peermentoring in online Facebook communities [25,26].

\subsection{Social media and sexual health discussion}

When dealing with information concerning sexual health, and HIV especially, the sharing of experiences through traditional channels can be difficult for individuals due to a reluctance to disclose sensitive personal information [23,27]. The perceived anonymity offered by social media allows users to discuss sexual health issues within online social circles with less fear of stigma [27-29]. Understanding the different kind of personal sexual health information that is shared online compared to in-person with clinicians and practitioners can serve as a valuable data source for tailoring future consumer health initiatives for HIV [23].

Studying social media trends allows discovery of factors useful in designing and implementing sexual health interventions that may be missed by traditional research [28]. An 
example of this includes determining the barriers to the increase in PrEP use among high risk MSM. Examples of the kinds of barriers discovered through analyzing social media discussion includes online ordering of PrEP, HIV and gay stigma, issues of perceived promiscuity of PrEP users, side effects of the medication, sexual risk compensation, experiences with poorly informed healthcare practitioners, and opinions on the pharmaceutical industry $[23,28,30]$.

Understanding online trends in sexual health and HIV discussions can allow researchers and consumer health providers to move beyond merely using social media as a way to disseminate information and instead leverage the interactive capabilities of the platforms to encourage participation and self-motivated interest in HIV prevention and sexual health among high risk MSM [11]. Encouraging active contribution of social media posts, compared to simple observation and endorsement, has shown to be an effective strategy in increasing HIV testing and sexual health awareness online [11]. Social media posts encourage people to share experiences and information and can trigger others to do the same, which in turn can increase the use of medication, help reduce anxiety, and to share rapid solutions to problems through impromptu virtual support groups [28].

An online presence from consumer health information providers delivering accurate and relevant information in response to these online expressions of need highlights the potential for powerful impacts on how social networks of high risk MSM discuss, understand, and engage with sexual health information and HIV prevention tools like PrEP.

\section{On-going and possible future research $\&$ the role of information professionals}

This review has uncovered numerous on-going and possible future research directions and the ways information professionals may be implicated in the improvement of online HIV consumer health information. 


\subsection{Challenges of tailoring community-level interventions for specific geographic, racial or}

social groups

Further research can be conducted into best practices concerning targeted HIV health information and the information behaviour and preferences of minority groups of MSM. There are indications that MSM of colour tend to be the most assortative in their sexual partners when using GSN apps and require more specific geographic and community-level interventions to achieve the best results [13]. Conversely, the explicit targeting of groups like young black MSM through GSN apps leads to potential stigmatization of an already high-risk sub-group of MSM $[9,17]$. Further study should be conducted to uncover the best messaging and information delivery strategies of online health information for specific vulnerable minority populations. This also includes those involved in transactional sex work, who tend to be at higher risk and are less likely to be reached through GSN apps or social media $[31,32]$.

\subsection{Application of information behaviour models to understand low PrEP uptake}

The slow uptake in PrEP use by high-risk MSM has revealed a knowledge-behaviour gap between awareness \& willingness to use PrEP compared with how many individuals actually take the drug regularly; studies range with results from one third to as high as 3 in 4 MSM being aware or willing to take PrEP, but with actual regular prescribed use of the drug being much lower (between 6-12\%) [33-36].

Research has been conducted to apply information behaviour modelling techniques (e.g., theory of planned behaviour; information-motivation-behavioural skills model) to analyze ways to improve consumer health information surrounding PrEP in order to increase uptake of the drug and improve HIV testing $[37,38]$. This research incorporates how information exposure, use of sexual health information, and experience accessing health care are influenced by personal 
perceptions of information relevance and contextualization of information through social interactions to motivate health decision making [37]. Use of information behaviour models, modifying existing models or the creation of new ones is an important direction for future research in addressing areas of concern in HIV prevention and treatment with appropriate consumer health resources and interventions.

\subsection{Development of information technology tools to support online HIV information}

The development of novel information technology tools in order to support online HIV consumer health is another research path holding great potential. Current research in this area includes: the development of an ontology-based FAQ query system for HIV/AIDS information [39], future versions of which could potentially be incorporated into GSN app sexual health information sections; continued research into social media data mining and monitoring to help make informed decisions and strategies concerning online HIV information materials [20,27,28]; and the creation of automated text summaries from health social media posts [40].

\subsection{Long term research}

Research gaps have been identified, including: a lack of large-scale longitudinal studies examining and clarifying the relationship between information behaviour changes and shifts in HIV/STI incidence in high risk MSM populations; a need for more comparative studies between MSM using GSN and those who are not and their differing information needs and behaviours; and the continuation and replication of existing studies in more diverse geographic and cultural regions [41]. Continuing collaboration between researchers, health information providers, and GSN app developers is essential in the continued evolution and success of this consumer health information medium [41]. On-going monitoring of changes in social media use and internet, mobile phone, and telecommunication technologies will also help ensure sexual health 
information professionals can most effectively deliver the highest quality and most useful information to high HIV-risk populations. 


\section{References}

1. Goedel WC, Mayer KH, Mimiaga MJ, Duncan DT. Considerable interest in pre-exposure prophylaxis uptake among men who have sex with men recruited from a popular geosocialnetworking smartphone application in London. Glob Public Health [Internet]. 2019 Jan 2 [cited 2020 Apr 14];14(1):112-21. Available from:

https://doi.org/10.1080/17441692.2017.1391859

2. Public Health Agency of Canada. HIV in Canada: 2018 surveillance highlights [Internet]. [Ottawa]: Government of Canada; 2019 Nov [cited 2020 Apr 15]. Available from: https:/www.canada.ca/en/public-health/services/publications/diseases-conditions/hiv-2018surveillance-highlights.html

3. Public Health Agency of Canada. Summary: estimates of HIV incidence, prevalence and proportion undiagnosed in Canada [Internet]. Ottawa (ON): Government of Canada. 2015 Nov [cited 2020 Apr 15]. Available from: https://www.canada.ca/en/publichealth/services/publications/diseases-conditions/summary-estimates-hiv-incidenceprevalence-proportion-undiagnosed-canada-2014.html

4. Grov C, Whitfield THF, Rendina HJ, Ventuneac A, Parsons JT. Willingness to take PrEP and potential for risk compensation among highly sexually active gay and bisexual men. AIDS Behav [Internet]. 2015 Dec 1 [cited 2020 Apr 14];19(12):2234-44. Available from: https://doi.org/10.1007/s10461-015-1030-1

5. Arkell C, Harrigan M. Oral pre-exposure prophylaxis (PrEP) [Internet]. [Toronto]: CATIE. 2019 [cited 2020 Apr 18]. Available from: https://www.catie.ca/en/factsheets/prevention/pre-exposure-prophylaxis-prep 
6. AVAC. Canada - PrEP Watch [Internet]. [New York]: AVAC. 2020 Apr 3 [cited 2020 Apr 20]. Available from: https://www.prepwatch.org/country/canada/

7. Schnall R, Travers J, Rojas M, Carballo-Diéguez A. eHealth interventions for HIV prevention in high-risk men who have sex with men: a systematic review. J Med Internet Res [Internet]. 2014 May 26 [cited 2020 Apr 14];16(5):e134. Available from: https://www.jmir.org/2014/5/e134/

8. Sun CJ, Stowers J, Miller C, Bachmann LH, Rhodes SD. Acceptability and feasibility of using established geosocial and sexual networking mobile applications to promote HIV and STD testing among men who have sex with men. AIDS Behav [Internet]. 2015 Mar [cited 2020 Apr 13];19(3):543-52. Available from: https://doi.org/10.1007/s10461-014-0942-5

9. Fields EL, Long A, Dangerfield DT, Morgan A, Uzzi M, Arrington-Sanders R, et al. There's an app for that: using geosocial networking apps to access young black gay, bisexual, and other MSM at risk for HIV. Am J Health Promot [Internet]. 2020 Jan [cited 2020 Apr 14];34(1):42-51. Available from https://doi.org/10.1177\%2F0890117119865112

10. Buttram ME, Kurtz SP. Representations of pre-exposure prophylaxis, informal antiretroviral medication use for HIV prevention, and diversion on geosocial networking apps among men who have sex with men. AIDS Behav [Internet]. 2019 Jul 15 [cited 2020 April 10];23(7):1790-6. 
11. Cao B, Liu C, Durvasula M, Tang W, Pan S, Saffer AJ, et al. Social media engagement and HIV testing among men who have sex with men in China: a nationwide cross-sectional survey. J Med Internet Res [Internet]. 2017 Jul 19 [cited 2020 Jan 26];19(7). Available from: https://www.ncbi.nlm.nih.gov/pmc/articles/PMC5541240/

12. Smith LW, Guy R, Degenhardt L, Yeung A, Rissel C, Richters J, et al. Meeting sexual partners through internet sites and smartphone apps in Australia: national representative study. J Med Internet Res [Internet]. 2018 Dec [cited 2020 Apr 5];20(12):e10683. Available from: https://www.jmir.org/2018/12/e10683/

13. Abuelezam NN, Reshef YA, Novak D, Grad YH, Seage GR III, Mayer K, et al. Interaction patterns of men who have sex with men on a geosocial networking mobile app in seven United States metropolitan areas: observational study. J Med Internet Res [Internet]. 2019 Sept [cited 2020 Apr 8];21(9):e13766. Available from: https://www.jmir.org/2019/9/e13766/

14. Hampel B, Kusejko K, Braun DL, Harrison-Quintana J, Kouyos R, Fehr J. Assessing the need for a pre-exposure prophylaxis programme using the social media app Grindr®. HIV Med [Internet]. 2017 Oct 16 [cited 2020 Mar 30];18(10):772-6. Available from: https://doi.org/10.1111/hiv.12521

15. Czarny HN, Broaddus MR. Acceptability of HIV prevention information delivered through established geosocial networking mobile applications to men who have sex with men. AIDS Behav [Internet]. 2017 Nov [cited 2020 Apr 12];21(11):3122-8. Available from: https://doi.org/10.1007/s10461-017-1743-4 
16. Gilbert M, Hottes TS, Kerr T, Taylor D, Fairley CK, Lester R, et al. Factors associated with intention to use internet-based testing for sexually transmitted infections among men who have sex with men. J Med Internet Res [Internet]. 2013 Nov 14 [cited 2020 Apr 2];15(11):e254. https://www.jmir.org/2013/11/e254/

17. Jones J, Carter B, Wilkerson R, Kramer C. Attitudes toward HIV testing, awareness of HIV campaigns, and using social networking sites to deliver HIV testing messages in the age of social media: a qualitative study of young black men. Health Educ Res [Internet]. 2019 Feb 1 [cited 2020 Apr 5];34(1):15-26. Available from: https://doi.org/10.1093/her/cyy044

18. Kesten JM, Dias K, Burns F, Crook P, Howarth A, Mercer CH, et al. Acceptability and potential impact of delivering sexual health promotion information through social media and dating apps to MSM in England: a qualitative study. BMC Public Health [Internet]. 2019 Sep 6 [cited 2020 Apr 8];19(1):1-9. Available from: https://doi.org/10.1186/s12889019-7558-7

19. Newcomb ME, Mongrella MC, Weis B, McMillen SJ, Mustanski B. Partner disclosure of PrEP use and undetectable viral load on geosocial networking apps: frequency of disclosure and decisions about condomless sex. J Acquir Immune Defic Syndr [Internet]. 2016 Feb 1 [cited 2020 Apr 8];71(2):200-6. Available from:

https://www.ncbi.nlm.nih.gov/pmc/articles/PMC4712713/ 
20. Grov C, Westmoreland D, Rendina HJ, Nash D. Seeing is believing? Unique capabilities of internet-only studies as a tool for implementation research on HIV prevention for men who have sex with men: a review of studies and methodological considerations. J Acquir Immune Defic Syndr [Internet]. 2019 Dec [cited 2020 Mar 30];82:S253-S260. Available from: https://pubmed.ncbi.nlm.nih.gov/31764261/

21. Magee JC, Bigelow L, DeHaan S, Mustanski BS. Sexual health information seeking online: a mixed-methods study among lesbian, gay, bisexual, and transgender young people. Health Educ Behav [Internet]. 2012 Jun 1 [cited 2020 Apr 4];39(3):276-89. Available from: https://doi.org/10.1177/1090198111401384

22. Nobles AL, Leas EC, Latkin CA, Dredze M, Strathdee SA, Ayers JW. \#HIV: Alignment of HIV-related visual content on Instagram with public health priorities in the US. AIDS Behav [Internet]. 2020 Jan 8 [cited 2020 Apr 18]; Available from: https://doi.org/10.1007/s10461-019-02765-5

23. McLaughlin ML, Hou J, Meng J, Hu C-W, An Z, Park M, et al. Propagation of information about preexposure prophylaxis (PrEP) for HIV prevention through Twitter. Health Commun [Internet]. 2016 Aug 2 [cited 2020 Apr 7];31(8):998-1007. Available from: https://doi.org/10.1080/10410236.2015.1027033

24. Schwartz J, Grimm J. PrEP on Twitter: information, barriers, and stigma. Health Commun [Internet]. 2017 Apr 3 [cited 2020 Apr 10];32(4):509-16. Available from: https://doi.org/10.1080/10410236.2016.1140271 
25. Ko N-Y, Hsieh C-H, Wang M-C, Lee C, Chen C-L, Chung A-C, et al. Effects of internet popular opinion leaders (iPOL) among internet-using men who have sex with men. J Med Internet Res [Internet]. 2013 Feb 25 [cited 2020 Apr 3];15(2):e40. Available from: https://www.jmir.org/2013/2/e40/

26. Patel VV, Ginsburg Z, Golub SA, Horvath KJ, Rios N, Mayer KH, et al. Empowering with PrEP (E-PrEP), a peer-led social media-based intervention to facilitate HIV preexposure prophylaxis adoption among young black and latinx gay and bisexual men: protocol for a cluster randomized controlled trial. JMIR Res Protoc [Internet]. 2018 Aug 28 [cited 2020 Apr 18];7(8). Available from: https://www.ncbi.nlm.nih.gov/pmc/articles/PMC6134229/

27. Christine K, Lazarus JV, Vassilis K. Mining social media for perceptions and trends on HIV pre-exposure prophylaxis. Stud Health Technol Inform [Internet]. 2019 [cited Apr 18 2020];959-963. Available from: http://ebooks.iospress.nl/publication/52131

28. Hannaford A, Lipshie-Williams M, Starrels JL, Arnsten JH, Rizzuto J, Cohen P, et al. The use of online posts to identify barriers to and facilitators of HIV pre-exposure prophylaxis (PrEP) among men who have sex with men: a comparison to a systematic review of the peer-reviewed literature. AIDS Behav [Internet]. 2018 Apr [cited 2020 Apr 16] ;22(4):1080-95. Available from: https://doi.org/10.1007/s10461-017-2011-3

29. Taggart T, Grewe ME, Conserve DF, Gliwa C, Roman Isler M. Social media and HIV: a systematic review of uses of social media in HIV communication. J Med Internet Res [Internet]. 2015 Nov 2 [cited 2020 Jan 26];17(11). Available from: https:/www.ncbi.nlm.nih.gov/pmc/articles/PMC4642795/ 
30. Franks J, Hirsch-Moverman Y, Loquere AS, Amico KR, Grant RM, Dye BJ, et al. Sex, PrEP, and stigma: experiences with HIV pre-exposure prophylaxis among New York City MSM participating in the HPTN 067/ADAPT study. AIDS Behav [Internet]. 2018 Apr [cited 2020 April 11];22(4):1139-49. Available from: https://doi.org/10.1007/s10461-0171964-6

31. Eaton LA, Driffin DD, Bauermeister J, Smith H, Conway-Washington C. Minimal awareness and stalled uptake of pre-exposure prophylaxis (PrEP) among at risk, HIVnegative, black men who have sex with men. AIDS Patient Care STDs [Internet]. 2015 Jul 28 [cited 2020 Apr 8];29(8):423-9. Available from: https://doi.org/10.1089/apc.2014.0303

32. Patel VV, Masyukova M, Sutton D, Horvath KJ. Social media use and HIV-related risk behaviors in young black and latino gay and bi men and transgender individuals in New York City: implications for online interventions. J Urban Health [Internet]. 2016 Apr [cited 2020 Apr 15];93(2):388-99. Available from: https://doi.org/10.1007/s11524-016-0025-1

33. Goedel WC, Halkitis PN, Greene RE, Hickson DA, Duncan DT. HIV risk behaviors, perceptions, and testing and preexposure prophylaxis (PrEP) awareness/use in Grindr-using men who have sex with men in Atlanta, Georgia. J Assoc Nurses AIDS Care [Internet]. 2016 Mar [cited 2020 Apr 15];27(2):133-42. Available from: https://doi.org/10.1016/j.jana.2015.11.005

34. Strauss BB, Greene GJ, Phillips G, Bhatia R, Madkins K, Parsons JT, et al. Exploring patterns of awareness and use of HIV pre-exposure prophylaxis among young men who have sex with men. AIDS Behav [Internet] 2017 May [cited 2020 Apr 17];21(5):1288-98. Available from: https://doi.org/10.1007/s10461-016-1480-0 
35. Schueler K, Ferreira M, Nikolopoulos G, Skaathun B, Paraskevis D, Hatzakis A, et al. Preexposure prophylaxis (PrEP) awareness and use within high HIV transmission networks. AIDS Behav. 2019 Jul;23(7):1893-903. Available from: https://doi.org/10.1007/s10461019-02411-0

36. Frankis JS, Young I, Lorimer K, Davis M, Flowers P. Towards preparedness for PrEP: PrEP awareness and acceptability among MSM at high risk of HIV transmission who use sociosexual media in four Celtic nations: Scotland, Wales, Northern Ireland and the Republic of Ireland: an online survey. Sex Transm Infect [Internet]. 2016 May 19 [cited 2020 Apr 14];92(4):279-85. Available from: http://dx.doi.org/10.1136/sextrans-2015052101

37. Meadowbrooke CC, Veinot TC, Loveluck J, Hickok A, Bauermeister JA. Information behavior and HIV testing intentions among young men at risk for HIV/AIDS. J Assoc Inf Sci Technol [Internet]. 2014 Feb 10 [cited 2020 Apr 13];65(3):609-20. Available from: https://doi.org/10.1002/asi.23001

38. Walsh JL. Applying the information-motivation-behavioral skills model to understand PrEP intentions and use among men who have sex with men. AIDS Behav [Internet]. 2019 Jul 15 [cited 2020 Apr 17];23(7):1904-16. Available from: https://doi.org/10.1007/s10461-0182371-3

39. Ayalew Y, Moeng B, Mosweunyane G. Experimental evaluation of ontology-based HIV/AIDS frequently asked question retrieval system. Health Informatics J [Internet]. 2019 Dec [cited 2020 Apr 16];25(4):1434-50. Available from: https://doi.org/10.1177\%2F1460458218775147 
40. Liu Y-H, Song X, Chen S-F. Long story short: finding health advice with informative summaries on health social media. Aslib J Inf Manag [Internet]. 2019 Nov 18 [cited 2020 Apr 14];71(6):821-40. Available from: https://doi.org/10.1108/AJIM-02-2019-0048

41. Zou H, Fan S. Characteristics of men who have sex with men who use smartphone geosocial networking applications and implications for HIV interventions: a systematic review and meta-analysis. Arch Sex Behav [Internet]. 2017 May [cited 2020 Apr 18];46(4):885-94. Available from: https://doi.org/10.1007/s10508-016-0709-3 